\title{
Apprentissage de règles de catégorisation dans le Trouble Développemental du Langage
}

\author{
Estelle DAUVISTER ${ }^{a, b}$ \& Christelle MAILLART ${ }^{a}$
}

${ }^{a}$ Research Unit for a life-Course perspective on Health \& Education, Département de logopédie, Université de Liège, Rue de l'Aunaie 30, 4000 Liège - Belgique. E-mails : i christelle.maillart@uliege.be

${ }^{\mathrm{b}}$ Fonds National pour la Recherche Scientifique - F.R.S.-F.N.R.S.

\begin{abstract}
Résumé
Les enfants ayant un trouble développemental du langage (TDL) présentent un vocabulaire réduit tant en quantité et qu'en qualité ainsi que des difficultés d'apprentissage de mots dont les causes restent méconnues. Cette recherche cible plus particulièrement le rôle des règles de catégorisation pour l'apprentissage lexical. A cette fin, une tâche de catégorisation implicite, comportant une règle, a été proposée à 17 enfants présentant un TDL et 17 enfants tout-venant d'âge scolaire. Cette tâche était suivie $\mathrm{d}$ 'une tâche de généralisation visant à appliquer la règle apprise à de nouvelles sous-catégories. Les résultats montrent que, globalement, les enfants présentant un TDL ne se distinguent pas de leurs pairs contrôles. Toutefois, lorsque les performances des enfants de moins de neuf ans et de neuf ans et plus sont examinées séparément, des différences de groupe émergent chez les plus jeunes. Ces résultats suggèrent des habiletés préservées dans l'apprentissage de règles simples, basées sur un critère perceptif, chez les enfants présentant un TDL plus âgés mais ces aptitudes se mettent en place plus tardivement que chez les enfants tout-venant.
\end{abstract}

Mots-clés : Trouble développemental du langage ; apprentissage de mots ; apprentissage de règles ; biais ; catégorisation

English title: Word learning and Developmental Language Disorder: Rule learning and generalization

Abstract: Children with developmental language disorder (DLD) show quantity and quality vocabulary limitations. These difficulties are associated with word learning difficulties that remain misunderstood. Our research focuses on these children's ability to learn categorisation rules from a statistical learning perspective. An implicit categorisation task, in which a rule could be formulated, was proposed to 17 schoolaged children with DLD and 17 typically developing children of the same age. This task was followed by a 
generalisation task, which measured the ability to apply the rule to new subcategories. The results showed globally preserved abilities for children with DLD. However, when a distinction was made between children under 9 and those aged 9 and over, differences emerged for the younger children. These results suggested preserved abilities in learning simple, perceptually based rules in older TDL children. However, it appeared that these abilities develop later than in the general population.

Keywords: Developmental language disorder; word learning; rule learning; bias; categorization 
Le trouble développemental du langage (TDL) se caractérise par des difficultés persistantes à développer et maîtriser la langue orale (Bishop et al., 2017). Il se manifeste de façon précoce et persiste jusqu'à l'adolescence et même l'âge adulte (Conti-Ramsden et al., 2012), entraînant des répercussions sur la vie quotidienne des personnes qui en sont affectées (Conti-Ramsden et al., 2018). Ces profils d'altérations langagières diverses en termes de domaines impactés et de sévérité, s'observent en l'absence de condition biomédicale associée ou de déficience intellectuelle (Bishop et al., 2017).

Parmi les difficultés langagières rencontrées, le domaine lexico-sémantique peut se trouver altéré (Leonard, 2014). Les enfants présentant un TDL présentent alors un vocabulaire limité en quantité de mots connus et richesse des informations sémantiques encodées (McGregor et al., 2013). Les capacités d'utilisation des mots en contexte se révèlent également plus faibles chez ces enfants comparativement à des enfants tout-venant. Charest et Skoczylas (2019) relèvent, par exemple, un emploi moins précis, plus inadéquat des termes connus compte tenu du contexte sémantique, et ce même lorsque les scores de vocabulaire des enfants présentant un TDL sont conformes à ce qui est attendu pour leur âge. Par ailleurs, la manière dont les enfants présentant un TDL organisent les informations sémantiques connues en mémoire semble plus diffuse que chez les enfants au développement typique (Brooks et al., 2017; Sheng \& McGregor, 2010a). L'apprentissage de mots constitue également une difficulté particulière pour les enfants présentant un TDL (Gray, 2003). Certains des facteurs influençant cet apprentissage ont pu être dégagés dans la métaanalyse de Kan et Windsor (2010) : ils concernent soit l'enfant lui-même (âge, niveau de langage réceptif, aptitudes intellectuelles non verbales), soit les tâches employées pour mesurer l'apprentissage (modalité de la tâche, type de mots à apprendre, nombre d'expositions aux nouveaux mots). Les difficultés d'apprentissage touchent par ailleurs autant les aspects phonologiques que sémantiques du lexique (Alt \& Plante, 2006; Jackson et al., 2021).

Outre leurs difficultés langagières, les enfants présentant un TDL présentent également des difficultés au niveau de mécanismes cognitifs plus généraux qui sont susceptibles d'intervenir dans l'apprentissage lexico-sémantique. Parmi ceux-ci, on identifie les ressources de traitement ainsi que les capacités d'apprentissage statistique qui soutiennent la détection de régularités dans les associations entre un mot et un référent d'une situation à l'autre. McMurray et collègues (2012) proposent que les premières interviennent au cours d'un temps situationnel et que les secondes sont mobilisées lors d'un temps développemental. Le temps situationnel concernerait alors l'étape au cours de laquelle l'enfant doit identifier le référent d'un mot en contexte d'ambiguïté. Le temps développemental concerne, quant à lui, I'apprentissage à proprement parler et réfère à l'encodage progressif de la bonne association entre le mot et le référent ainsi qu'à l'ajout de caractéristiques du référent à la représentation formée en mémoire. Selon ce modèle, l'apprentissage s'effectue au fur et à mesure des rencontres entre l'association du mot et de son référent, il est dit inter-situationnel (McMurray et al.,2012).

Des difficultés sont relevées pour les deux types de processus chez les enfants présentant un TDL : ils présentent une limitation des ressources de traitement (Im-Bolter et al., 2006) et des déficits au niveau des aptitudes d'apprentissage statistique. L'apprentissage statistique fait référence à l'aptitude à détecter et extraire des régularités, des corrélations ou cooccurrences d'indices, dans l'environnement langagier et non 
langagier (Aslin, 2017). Les enfants présentant un TDL manifestent des aptitudes d'apprentissage statistique plus faibles que celles de leurs pairs dans de nombreux domaines, à la fois langagiers et non langagiers, et dans de nombreuses tâches (Lammertink et al., 2017; Lum et al., 2014; Obeid et al., 2016). Les études montrent ainsi que les enfants présentant un TDL segmentent plus difficilement que leurs pairs tout-venant le flux de parole en mots (Evans et al., 2009; Haebig et al., 2017). Un taux plus important d'exposition au flux de parole ne leur permet pas de rattraper leurs pairs (Evans et al., 2009). Les aptitudes d'apprentissage statistique, évaluées à l'aide de tâches de segmentation de mots, sont par ailleurs liées aux caractéristiques phonologiques, mais pas sémantiques, du vocabulaire des enfants présentant un TDL (Mainela-Arnold \& Evans, 2014). La majeure partie des tâches employées impliquent la détection de régularités séquentielles. Or, selon le modèle de McMurray et al. (2012) et l'idée d'un apprentissage de mots trans-situationnel, les régularités statistiques distributionnelles pourraient s'avérer davantage pertinentes pour apprendre les aspects sémantiques du vocabulaire (Lammertink et al., 2017) que les régularités séquentielles. Ces régularités se répartiraient sur plusieurs rencontres avec un mot et son référent par exemple.

Le résultat de l'apprentissage statistique peut être spécifique, puisqu'il dépend des caractéristiques de l'environnement, ou plus abstrait et prendre la forme d'une règle (Aslin \& Newport, 2012; Reeder et al., 2013). Apprentissage spécifique et apprentissage de règles constituent alors les extrémités opposées d'un même continuum. L'apprentissage de règles concernerait l'abstraction de propriétés plus globales et communes à plusieurs items, plutôt que spécifiques à un seul d'entre eux, comme les propriétés et la structure des catégories sémantiques par exemple (Aslin \& Newport, 2012). Il est de cette façon lié à la notion de généralisation, puisqu'il permet d'appliquer un schéma abstrait à des items non rencontrés qui partagent des propriétés communes avec ceux déjà rencontrés (Aslin, 2017). Enfin, le degré d'abstraction résultant de l'apprentissage statistique pourrait être lié aux indices contextuels qui accompagnent l'objet de l'apprentissage (Aslin \& Newport, 2012; Reeder et al., 2013). Par exemple, des indices contextuels plus variables mèneraient à un apprentissage davantage décontextualisé et donc à la formulation de règles plutôt que d'apprentissages spécifiques.

Smith et collègues (2002) proposent un modèle d'apprentissage et de généralisation lexicale en quatre étapes rendant compte de ce continuum entre apprentissage spécifique et apprentissage de règles. Dans ce modèle, la première étape renvoie à l'apprentissage d'une association spécifique entre un mot et un référent. Par exemple, l'enfant apprend que le mot « tasse » renvoie à la tasse dans laquelle il boit son lait le matin. Ensuite, au cours de la deuxième étape, l'enfant apprend la structure des catégories spécifiques. II apprend quelles sont les propriétés importantes de la catégorie « tasse » : elle a une anse et elle présente une forme creuse. Cette étape constitue celle de la généralisation de premier ordre car elle permet à l'enfant d'appliquer un terme appris à tous les référents qui partagent les propriétés identifiées comme pertinentes pour ce mot. La troisième étape, quant à elle, fait référence à la généralisation de second ordre. Au cours de celle-ci, l'enfant qui a appris la structure de plusieurs catégories spécifiques formule une règle plus générale qui tient compte des propriétés redondantes entre ces catégories. Par exemple, il aura appris, en plus de la catégorie "tasse ", que la catégorie " chaise " regroupe des objets à quatre pieds, avec une assise et un dossier. II pourra alors s'apercevoir que les objets sont souvent nommés selon leur forme générale et le 
formuler sous forme de règle. Enfin, la quatrième étape correspond à la mobilisation des règles acquises dans sa vie quotidienne. L'enfant y apprend davantage de mots, à un rythme plus rapide (Smith et al., 2002).

Le biais pour la forme constitue un exemple de ces règles de généralisation de second ordre. II se manifeste de façon précoce dans le développement (Smith, 2003) et peut être induit chez le très jeune enfant par entraînement (Smith et al., 2002). Il se traduit par une tendance à appliquer un mot à un référent qui partage des caractéristiques de forme avec les exemplaires déjà rencontrés de ce mot. Chez l'enfant présentant un TDL, le biais pour la forme se met en place plus tardivement que dans le développement typique. Lorsqu'ils doivent appliquer un mot à un nouvel exemplaire (extension de mot), les enfants présentant un TDL d'âge préscolaire se basent aussi souvent sur des critères de forme que de substance ou de couleur, là où leurs pairs de même âge manifestent un préférence plus systématique pour la forme (Collisson et al., 2015). Le biais pour la forme peut, par contre, être mobilisé par les enfants présentant un TDL d'âge scolaire. Ces derniers semblent même en faire une utilisation excessive, puisqu'ils persévèrent sur le critère de forme même pour des catégories définies par d'autres caractéristiques. Ces enfants présentent en effet des difficultés pour des catégories de substance et de relations spatiales notamment, alors que les enfants tout-venant de même âge peuvent diriger leur attention vers les caractéristiques pertinentes de ces catégories également (Krzemien et al., 2021). Le biais pour la forme s'installe donc plus tardivement chez les enfants présentant un TDL, qui semblent le sur-généraliser une fois qu'il a été acquis.

Dans le TDL, les mécanismes d'apprentissage statistique ont été mis en lien avec les capacités d'apprentissage des aspects phonologiques des mots, mais les caractéristiques sémantiques ont été peu étudiées. Plus spécifiquement, l'apprentissage statistique séquentiel, mais pas distributionnel comme pourrait l'être un apprentissage trans-situationnel, a été évalué chez les enfants présentant un TDL. Par ailleurs, les résultats exposés suggèrent que les enfants présentant un TDL présentent des difficultés à apprendre des règles de catégorisation pour soutenir leurs apprentissages lexicaux et la généralisation de nouveaux mots. Ces résultats se limitent toutefois à l'étude du biais pour la forme, mais ne renseignent pas sur les habiletés plus globales des enfants présentant un TDL à formuler une règle, ou un biais, qu'ils puissent appliquer à de nouveaux mots.

\section{Objectifs et hypothèses de l'étude}

Notre étude a pour objectif principal de déterminer les capacités d'apprentissage d'une règle de catégorisation chez enfants présentant un TDL, à un niveau de généralisation de premier ordre à partir de régularités statistiques trans-situationnelles. Nous avons également étudié les capacités des enfants présentant un TDL à généraliser la règle apprise, à un niveau de généralisation de second ordre. A cette fin, nous avons proposé à des enfants présentant un TDL et des enfants tout-venant de même âge chronologique une tâche de catégorisation implicite pour évaluer la généralisation de premier ordre et une tâche de généralisation à d'autres catégories de même structure pour évaluer la généralisation de second ordre.

Plusieurs hypothèses peuvent être formulées. Premièrement, les enfants présentant un TDL pourraient apprendre moins efficacement une règle de catégorisation basée sur un critère que leurs pairs tout-venant. Plus précisément, les enfants présentant un TDL devraient parvenir à identifier la règle de 
catégorisation, mais seulement à condition d'avoir bénéficié d'un nombre accru d'exemplaires. L'augmentation du nombre d'exemplaires nécessaires suggérerait alors des difficultés d'apprentissage statistique distributionnel chez les enfants présentant un TDL.

Deuxièmement, les enfants présentant un TDL pourraient ne pas parvenir à formuler une règle de catégorisation à un degré d'abstraction aussi élevé que leurs pairs tout-venant. Pour réussir notre tâche de généralisation, les participants doivent parvenir à formuler une règle abstraite qui doit pouvoir s'appliquer à de nouvelles catégories de même structure. Les enfants présentant un TDL pourraient généraliser moins efficacement la règle apprise à de nouvelles catégories, ce qui se marquerait par des scores plus faibles à la tâche de généralisation et traduirait l'acquisition d'une règle plus spécifique ou moins abstraite.

\section{Méthodologie}

La recherche menée a reçu l'aval du comité d'éthique local (réf. 1718-54). Un consentement écrit a été obtenu de la part des parents ou tuteurs légaux des participants. Un consentement oral ou écrit, selon les capacités de l'enfant, a également été obtenu pour chacun des enfants.

\section{Participants}

Deux groupes de participants ont été recrutés. Le premier était constitué de 17 enfants ayant reçu un diagnostic de TDL et scolarisés dans l'enseignement spécialisé de la partie francophone de Belgique. Ces enfants étaient âgés de 6 ans 6 mois à 11 ans 11 mois et ne présentaient pas de conditions biomédicales associées aux troubles langagiers. Un impact fonctionnel peut être constaté, puisque ces enfants bénéficient d'un cursus scolaire adapté. Une évaluation langagière a été proposée à chaque enfant pour confirmer le diagnostic et définir le profil de ce groupe. Les aptitudes phonologiques en production ont été évaluées à I'aide de la tâche de répétition de mots de la batterie E.L.O. (Evaluation du Langage Oral, Khomsi, 2001). Le vocabulaire a été évalué à l'aide de l'épreuve de dénomination de l'E.L.O. (Khomsi, 2001) en production, et de l'Echelle de Vocabulaire en Images Peabody (Dunn et al., 1993) en compréhension. Pour évaluer la morphosyntaxe, une épreuve de complétion d'énoncés (E.L.O., Khomsi, 2001) a été proposée en production et une épreuve de désignation d'images (Epreuve de Compréhension Syntaxico-Sémantique, Lecoq, 1996) a évalué la compréhension. Les enfants présentant un TDL présentent tous au moins deux scores sous le percentile 10 des enfants de leur âge ou de leur niveau scolaire. Leur profil langagier se caractérise par des scores déficitaires (sous le percentile 3 ) aux niveaux de la phonologie et de la morphosyntaxe. Les scores de vocabulaire se situent, quant à eux, dans les normes pour un grand nombre d'enfants, mais restent inférieurs à ceux des enfants contrôle. Enfin, les aptitudes intellectuelles non verbales ont été évaluées à l'aide de l'échelle Non Verbale d'intelligence de Weschler (WNV, Weschler \& Naglieri, 2009) et sont supérieures au critère de déficience intellectuelle pour chaque enfant du groupe $(\min .=82, \max .=110, m=93.04$, e- $t=$ 8.22).

Le second groupe, groupe contrôle, était constitué de 17 enfants tout-venant appariés en âge chronologique et niveau d'aptitudes intellectuelles non verbales avec les enfants présentant un TDL. Pour l'âge, un écart de plus ou moins trois mois était toléré, alors que pour le QI non verbal, l'écart toléré était de plus ou moins 7 points $(\min .=85, \max .=110, m=94$, e-t $=6.8$ ). Les enfants étaient âgés de 6 ans 7 mois à 
11 ans 11 mois et étaient scolarisés dans l'enseignement ordinaire de la même région. Sur la base d'un questionnaire parental, aucune inquiétude n'a été relevée par rapport au langage ou à d'autres conditions biomédicales. Une évaluation langagière similaire à celle des enfants présentant un TDL a confirmé l'absence de difficultés langagières chez ces enfants, qui obtiennent des scores supérieurs au percentile 10 des enfants de même âge ou niveau scolaire pour chacun des tests employés. Les caractéristiques langagières et cognitives des deux groupes sont synthétisées dans le Tableau 1.

\section{[Insérer le Tableau 1]}

\section{Matériel}

Une tâche à choix forcé en deux parties a été proposée aux participants. La première partie concernait I'apprentissage de la règle, au cours d'une tâche de catégorisation, et la seconde concernait la généralisation de cette règle, par son application à de nouvelles catégories.

Pour la tâche de catégorisation, deux catégories d'insectes ont été créées. Toutes deux répondaient à une même structure et étaient définies par le nombre de doigts des insectes : trois pour la catégorie des 'mopi' et cinq pour la catégorie des 'dufan'. Seule cette caractéristique était pertinente pour déterminer l'appartenance à la catégorie, les autres étant non systématiquement associées à une catégorie ou à l'autre. Chaque planche de la tâche se composait d'un exemplaire d'une des deux catégories associé à un non-mot étiquetant la catégorie ainsi que de deux possibilités de réponse (la cible et le distracteur). Le distracteur était, pour chaque essai, un exemplaire de la deuxième catégorie à apprendre. La tâche comportait maximum 40 items (20 par catégorie). Les non-mots sont de structure consonne-voyelle-consonne-voyelle et respectent les règles phonotactiques du français. La généralisation de premier ordre testée consiste en l'apprentissage d'une règle de type "Les 'mopi' sont des 'mopi' parce qu'ils ont trois doigts et les 'dufan' sont des 'dufan' parce qu'ils ont cinq doigts ».

Une tâche similaire comportant d'autres catégories d'insectes, définies par le même critère, constitue la tâche de généralisation de la règle apprise similaire. Elle permet d'évaluer la généralisation de second ordre, soit la capacité à étendre les catégories précédemment apprises. La règle à formuler était de type « les catégories d'insectes sont définies par le nombre de doigts ». Un total de 15 items composait cette tâche. Ils étaient répartis en proportions équivalentes entre trois nouvelles catégories (les insectes à un, deux et quatre doigts) associées à de nouveaux non-mots. Un exemplaire de chacune des catégories créées est présenté dans la Figure 1.

\section{[insérer la Figure 1]}

\section{Procédure}

La tâche a été programmée et administrée à l'aide du logiciel OpenSesame (Mathôt et al., 2012) sur un ordinateur portable avec un écran de 15 pouces. Elle était proposée sous forme de jeu dans lequel une tempête a éclaté et a mélangé tous les insectes sans qu'ils puissent retrouver leurs maisons respectives. L'enfant devait réaliser une tâche de catégorisation puis une tâche de généralisation. Dans la première, il devait catégoriser les insectes sur la base de leur nom (" mopi » ou « dufan »). Afin de lui permettre d'ajuster 
ses réponses, l'enfant était averti qu'en cas d'erreur, un feedback visuel lui serait fourni pour qu'il puisse identifier la bonne réponse. La tâche de catégorisation prenait fin soit après les 40 items, soit lorsque cinq bonnes réponses consécutives étaient fournies (critère d'apprentissage, supérieur au niveau du hasard). La tâche de généralisation était ensuite proposée en lui demandant de poursuivre l'exercices avec d'autres insectes. Un exemple d'item pour chacune des deux phases est proposé dans la Figure 2.

\section{[insérer la Figure 2]}

Les réponses, non verbales, étaient fournies à l'aide de touches-réponses sur le clavier qui se situaient du côté de l'insecte qu'il voulait sélectionner. Des repères visuels, proposés sous forme de gommettes de couleur, servaient de soutien aux touches-réponses.

\section{Traitement des données}

Afin de déterminer dans quelle mesure les enfants présentant un TDL parviennent à apprendre la règle de catégorisation, le nombre d'essais nécessaires pour atteindre le critère d'apprentissage ont été comptabilisés. Le succès de la généralisation était déterminé par le nombre de réponses correctes pour la tâche de généralisation. Etant donné qu'aucune différence significative ne peut être notée sur les temps de réponse, ces données ne seront pas exploitées ici.

Les résultats ont été traités en trois temps. Tout d'abord, nous avons comparé les performances des deux groupes en termes de nombre d'essais nécessaires pour la tâche de catégorisation. Les conditions de normalité des données n'étant pas respectées, et nos effectifs différant d'un groupe à l'autre, des tests $U$ de Mann-Whitney ont été employés. La même procédure a ensuite été appliquée aux scores à la tâche de généralisation. Enfin, compte tenu de la grande variabilité des scores de catégorisation, de la différence de six à sept points relevée qualitativement au niveau du nombre d'essais nécessaires pour atteindre le critère d'apprentissage, et de la nature développementale et retardée de l'apparition du biais pour la forme dans le TDL, nous avons poursuivi nos analyses en scindant chacun des groupes en deux selon l'âge des participants.

Nous avons considéré l'âge intermédiaire, de 9 ans, comme le critère nous permettant de séparer les groupes en deux. Le groupe d'enfants jeunes se compose des enfants de 6 ans à 8 ans 11 mois et le groupe d'enfants âgés se compose des enfants de 9 ans à 11 ans 11 mois. La tranche d'âge du groupe d'enfants jeunes coïncide par ailleurs avec la tranche d'âge au cours de laquelle une réorganisation lexico-sémantique est proposée (Sloutsky et al., 2015). La distinction selon le profil langagier a par ailleurs été conservée, de sorte que nous avons comparé les performances des jeunes enfants présentant un TDL à celles des jeunes enfants contrôle et pareillement pour les plus âgés. Suite à la scission, nos groupes d'enfants jeunes se composaient chacun de huit enfants et nos groupes d'enfants âgés de neuf enfants. A nouveau, des tests $U$ de Mann-Whitney ont été utilisés pour comparer nos groupes en raison de violations des conditions d'application du test $t$ de Student pour échantillons indépendants. Ces tests non paramétriques semblent également plus adaptés aux effectifs particulièrement réduits obtenus suite à la scission. 


\section{Résultats}

Apprentissage d'une règle : généralisation de premier ordre

Les résultats du test $U$ de Mann-Whitney sont non significatifs et suggèrent un nombre d'essais moyen similaire pour les deux groupes $(U(32)=99.00, p=.12)$. Ainsi, les enfants tout-venant et présentant un TDL avaient besoin de respectivement $13,41( \pm 9,23)$ et $21,41( \pm 13,33)$ essais en moyenne pour atteindre le critère fixé.

\section{Apprentissage d'une règle : généralisation de second ordre}

Les résultats du test de Mann-Whitney indiquent une absence de différence significative entre les groupes $(U(32)=164.50, p=.477)$ et suggèrent des performances similaires en généralisation. En effet, les enfants présentant un TDL fournissent en moyenne $11,29( \pm 4,24)$ réponses correctes et les enfants contrôle 12,71 $( \pm$ $3,14)$.

\section{Analyse des performances par groupe d'âge}

\section{Apprentissage d'une règle : généralisation de premier ordre}

Pour les enfants plus âgés, les résultats indiquent une absence de différence significative entre les enfants présentant un TDL et les enfants contrôle pour la tâche de catégorisation $(U(16)=31.00, p=.425)$. Les enfants contrôle avaient besoin de 14,66 $( \pm 10,56)$ essais en moyenne pour atteindre le critère d'apprentissage, et les enfants présentant un TDL de 19,33 ( $\pm 11,66)$ essais. Pour les enfants plus jeunes, les résultats indiquent une différence significative entre les deux groupes pour la tâche de catégorisation $(U(14)=13.00, p=.05)$ avec une taille d'effet moyenne ( $d$ de Cohen $=-0.59$ ). Les jeunes enfants présentant un TDL ont besoin de davantage d'essais ( $m=28,12$, et $=14,30)$ pour apprendre une règle de catégorisation à un critère que leurs pairs tout-venant $(m=12,00$, et $=8,02)$.

Contrairement aux enfants plus âgés, certains enfants présentant un TDL de moins de neuf ans échouent par ailleurs à atteindre le critère d'apprentissage. En effet, seuls quatre enfants sur huit atteignent ce critère, alors que tous les enfants contrôle l'atteignent. Ainsi, lorsque l'on compare la proportion d'enfants jeunes qui parviennent à apprendre la règle de généralisation dans les deux groupes à l'aide d'un test de Chicarré d'indépendance, les résultats indiquent une différence significative $\left(\chi^{2}=5.33, p=.02\right)$.

\section{Apprentissage d'une règle : généralisation de second ordre}

Comme pour la généralisation de premier ordre, le test de Mann-Whitney pour la tâche de généralisation révèle une absence de différence significative entre les enfants présentant un TDL et les enfants contrôle les plus âgés $(U(16)=30.00, p=.354)$. Les enfants présentant un TDL réussissent en moyenne $12,44( \pm 3,94)$ essais, et les enfants contrôle en réussissent $11,22( \pm 3,70)$ sur 15 .

Des différences significatives émergent pour les enfants plus jeunes $(U(14)=53.00, p=.024)$ avec une taille d'effet moyenne $(d$ de Cohen $=0.66$ ), suggérant que les enfants présentant un TDL plus jeunes 
parviennent moins bien à généraliser la règle apprise à de nouvelles catégories de même structure que leurs pairs tout-venant. Ces enfants obtiennent un score moyen de $9,37( \pm 3,96)$, parfois à peine supérieur au niveau du hasard, alors que leurs pairs réalisent un score quasi maximal avec une moyenne de $14,37( \pm 0,92)$. La proportion d'enfants présentant un TDL qui parviennent à généraliser la règle est également inférieure à celle des enfants tout-venant $\left(\chi^{2}=7.273, p=.007\right)$, qui atteignent tous des scores maximaux ou presque. Notons que ces résultats doivent être considérés avec prudence compte tenu des faibles effectifs, mais indiquent qu'alors que tous les enfants tout-venant parviennent à généraliser la règle apprise, la moitié des enfants présentant un TDL n'y parvient pas. Les performances à la tâche de généralisation révèlent par ailleurs que soit la règle a été apprise et peut très bien être généralisée, soit elle ne l'a pas été et les enfants obtiennent une performance proche du niveau du hasard. Ainsi, parmi les cinq enfants présentant un TDL qui échouent à la tâche de généralisation, trois n'atteignaient pas le critère d'apprentissage dans la phase précédente.

\section{Discussion}

Nous avons étudié les capacités d'apprentissage d'une règle de catégorisation et de généralisation de la règle chez les enfants présentant un TDL d'âge scolaire. Nous avions formulé l'hypothèse de difficultés par rapport à leurs pairs de même âge chronologique, qui se seraient manifestées par un nombre d'essais plus important avant d'atteindre le critère d'apprentissage et des scores plus faibles pour la généralisation. Lorsque nous avons considéré les groupes dans leur ensemble, nos résultats ont révélé une absence de différence significative à la fois pour la tâche de catégorisation, c'est-à-dire l'apprentissage de la règle, et pour la tâche de généralisation, c'est-à-dire pour l'application de la règle à d'autres catégories de même structure. Ces résultats infirment nos hypothèses et suggèrent des habiletés d'apprentissage de régularités statistiques distributionnelles préservées chez les enfants présentant un TDL. En accord avec le modèle de Smith et al. (2002), les enfants présentant un TDL de notre groupe se sont montrés capables d'effectuer des généralisations de premier ordre (étape 2) et des généralisations de second ordre (étape 3 ).

Compte tenu de l'âge des enfants qui constituent nos groupes de participants, les résultats obtenus de manière globale concordent avec les observations croisées des études de Collisson et al. (2015) et Krzemien et al. (2021) ayant trait au biais pour la forme. Tout comme ces auteurs montraient que le biais pour la forme était absent chez les enfants présentant un TDL d'âge préscolaire, mais présent chez les enfants d'âge scolaire, il est possible que les enfants de notre groupe aient développé des aptitudes d'apprentissage de règles suffisantes compte tenu de leur âge.

Examiner nos résultats selon l'âge des participants nous a mené à les nuancer. Les aptitudes d'apprentissage et de généralisation d'une règle de catégorisation se sont en effet révélées similaires à celles d'enfants de même âge pour les enfants de neuf ans et plus, mais se sont par contre avérées inférieures chez les enfants présentant un TDL de moins de neuf ans. Ces résultats concordent avec les données qui observent qu'un plus grand nombre d'exemplaires sont nécessaires pour que les enfants présentant un TDL parviennent à acquérir de nouveaux mots (Gray, 2003, 2004). Ils sont également en accord avec les données issues de la 
méta-analyse de Kan et Windsor (2010), qui relatent un écart de performances plus important par rapport aux enfants tout-venant chez les enfants présentant un TDL plus jeunes que chez les TDL enfants plus âgés.

Plutôt que d'envisager les aptitudes d'apprentissage de règle et de généralisation de cette règle comme une compétence préservée chez les enfants présentant un TDL, ces aptitudes semblent donc pouvoir s'envisager dans une perspective développementale. II est possible que les enfants présentant un TDL développent de manière progressive leur aptitude à acquérir des biais et règles de catégorisation et que les données relatives au biais pour la forme traduisent la dynamique du développement de cette capacité.

Deux facteurs sont susceptibles d'expliquer la différence de résultats obtenus entre les deux groupes d'âge. Tout d'abord, il est possible que celle-ci soit due à l'implication des ressources de traitement dans l'apprentissage lexical. Si les ressources de traitement sont impliquées dans l'apprentissage de mots, et notamment dans la phase de sélection du référent (McMurray et al., 2012), il est possible qu'une différence de maturation au niveau de ces ressources, et des fonctions exécutives en particulier, puisse rendre compte d'une partie des différences observées selon l'âge. Les enfants présentant un TDL présentent des difficultés au niveau des ressources de traitement, dont les fonctions exécutives et la mémoire de travail, qui sont toujours manifestes à l'âge scolaire (Im-Bolter et al., 2006; Pauls \& Archibald, 2016; Vugs et al., 2013). Chez l'enfant présentant un TDL d'âge préscolaire, les fonctions exécutives, telle l'inhibition, et les aptitudes de mémoire de travail auditivo-verbale apparaissent liées à l'apprentissage de mots (Kapa \& Erikson, 2020). Les résultats chez l'enfant plus grand, d'âge scolaire, semblent par contre moins consensuels : alors que certains mettent en évidence des liens entre la mémoire de travail et les aptitudes d'apprentissage de mots (Jackson et al., 2016), d'autres obtiennent des résultats contraires (Gray, 2006; Mainela-Arnold et al., 2010). Alors que l'on sait que les fonctions exécutives se développent avec l'âge, l'évolution des ressources de traitement dans le TDL et leur interaction avec l'apprentissage de mots dans le TDL reste donc sujet à controverse. Toutefois, les résultats existants pourraient laisser supposer que ces fonctions exécutives impactent différemment les apprentissages des enfants plus jeunes et plus âgés, et qu'il s'agisse d'un facteur qui contribue de facto aux différences que nous avons observées. Des difficultés dans la première étape d'apprentissage de mots, l'étape situationnelle, pourraient alors conduire à un apprentissage cross-situationnel plus lent.

Ensuite, il est possible que les différences observées puissent être expliquées par la nature de la règle à apprivoiser. En effet, cette règle peut être définie par un seul critère, de nature perceptive, et peut donc être considérée comme relativement simple à identifier. A cet égard, il semble que les enfants présentant un TDL présentent davantage de facilités pour l'acquisition des catégories concrètes et définies perceptivement, telles les catégories d'objets, par rapport à d'autres types de catégories. C'est par exemple ce que montrent Krzemien et al. (2021), qui rapportent des difficultés pour les catégories de substances ou définies par la relation entre les éléments qu'elles incluent. Ceci rejoint également le constat d'une plus grande difficulté chez les enfants présentant un TDL à maîtriser la catégorie des verbes (Sheng \& McGregor, 2010b; Thordardottir \& Weismer, 2002), elle-même relationnelle et plus abstraite par nature. II semble donc que tous les types de mots et de catégories ne soient pas maîtrisés de manière équivalente pour les enfants présentant un TDL que pour les enfants tout-venant de même âge. Si les enfants présentant un TDL d'âge scolaire sont parvenus à maîtriser le biais pour la forme (Krzemien et al., 2021), il est possible que notre 
groupe d'enfants présentant un TDL plus âgés ait été capable d'apprendre la règle impliquée dans notre étude, qui s'apparente elle aussi à une règle basée sur un critère perceptif. En revanche, nos résultats n'impliquent pas que les enfants présentant un TDL manifestent de telles aptitudes pour tous les types de critères et de catégories existants, et ne permettent pas de déterminer dans quelle mesure des règles plus complexes ou basées sur des critères relationnels représentent un challenge plus important.

Dans la même idée, nos résultats concordent également avec l'observation de scores de vocabulaire dans la moyenne attendue pour les enfants présentant un TDL, malgré les difficultés d'apprentissage et d'usage de mots constatées (Charest \& Skoczylas, 2019). Une majorité de tâches permettant d'évaluer le niveau de vocabulaire des enfants se centre en effet principalement sur des catégories de mots concrets, imageables, ou qui peuvent se définir par des caractéristiques perceptives, mais moins sur des catégories relationnelles (Krzemien et al., 2020). Si, comme nos résultats et d'autres le suggèrent, certaines aptitudes d'apprentissage de mots se mettent en place progressivement avec l'âge, et que les enfants présentant un TDL plus âgés sont parvenus à maîtriser les principes d'acquisition des catégories perceptives ou concrètes, il se peut que ces mesures, tout comme celle de notre tâche, ne soient pas suffisamment sensibles pour permettre d'appréhender pleinement la nature des difficultés lexicales des enfants présentant un TDL. II pourrait dès lors être intéressant de contraster nos résultats avec une condition d'apprentissage dont la règle est plus complexe à acquérir, et impliquerait par exemple une règle définie par une relation d'éléments.

Enfin, nous souhaitons revenir brièvement sur les résultats qui concernent notre tâche de généralisation, et donc l'étape de généralisation de second ordre. Dans notre étude, les enfants qui parviennent à apprendre la règle de catégorisation lors de la première phase parviennent très bien à la généraliser lors de la seconde phase. Les performances des enfants s'apparentaient en effet à l'idée d'un phénomène de " tout ou rien »: ou la règle était acquise et pouvait être généralisée presque parfaitement, ou elle ne l'était pas et les scores approchaient le niveau du hasard. Il est possible que ces scores plafond reflètent le renforcement progressif de l'apprentissage des enfants. Cependant, il serait étonnant que ce facteur seul permette de gommer toute variation interindividuelle dans les aptitudes de généralisation des enfants.

Nous dégageons deux pistes d'explication pour ce phénomène de " tout ou rien ". Premièrement, l'augmentation de la variabilité du critère pertinent, le nombre de doigts, avec de nouvelles catégories pourrait avoir permis aux enfants d'effectuer la tâche plus aisément. Reeder et al. (2013) proposaient que le niveau d'abstraction d'un apprentissage était susceptible de varier en fonction du contexte d'apparition de l'objet de l'apprentissage. Par ailleurs, Perry et al. (2010) mettaient en évidence un effet facilitateur de la variabilité des exemplaires proposés lors de la familiarisation sur les capacités d'apprentissage et de généralisation de mots de jeunes enfants tout-venant. Cet effet facilitateur se retrouve également chez les enfants présentant un TDL (Aguilar et al., 2018). D'un point de vue clinique, il est possible qu'augmenter la variabilité du contexte d'apprentissage représente une piste intéressante pour la planification des interventions. De futures études pourraient s'attacher à observer dans quelle mesure ce facteur peut effectivement représenter une condition facilitante pour les apprentissages dans le TDL. 
Deuxièmement, il est possible que les critères spécifiques de chaque catégorie proposée, à savoir un nombre de doigts précis, fassent eux-mêmes partie d'une catégorie fortement ancrée dans les connaissances des enfants testés. Ils font en effet tous référence à des nombres compris entre un et cinq, et les habiletés numériques et de comptage pour ces nombres sont souvent bien développées à l'âge scolaire. Ainsi, la caractéristique de nombre pourrait avoir été particulièrement facile à abstraire à un niveau de généralisation de second ordre, étant donné que la catégorie des nombres est probablement déjà bien formée dans le réseau de connaissances des enfants. En complément à l'examen de la variabilité, examiner le contraste de performances à des degrés de connaissance ou de variabilité divers au niveau des critères, et non des caractéristiques spécifiques, constitue une perspective intéressante pour cerner les compétences préservées ou non des enfants présentant un TDL.

\section{Limites}

Notre étude comporte des limitations méthodologiques. Tout d'abord, nos échantillons de participants sont restreints, puisqu'ils comportent 17 enfants par groupe pour les résultats globaux, et 8 et 9 enfants par groupe pour les enfants plus jeunes et plus âgés respectivement. Les conclusions que l'on peut tirer de nos résultats sont en conséquence limités et doivent faire l'objet de prudence. II serait nécessaire de reproduire les résultats obtenus sur de plus grands échantillons, particulièrement en ce qui concerne les différences observées selon l'âge des enfants.

Ensuite, il se peut que la tâche ait été particulièrement simple pour des enfants de l'âge ciblé. II pourrait être pertinent d'envisager des situations d'apprentissage dont les paramètres sont plus variés, et notamment d'observer les capacités d'apprentissage de règles pour des mots ou catégories définis par d'autres types de critères, ou une intrication de critères rendant la tâche plus complexe.

Enfin, il pourrait s'avérer intéressant d'examiner plus directement et plus en profondeur les liens entre les capacités d'apprentissage dans ce type de tâche et les deux types de processus mobilisés dans le modèle d'apprentissage de mots de McMurray et al. (2012).

\section{Conclusions}

En conclusion, nos résultats suggèrent que, considérés comme un seul groupe, les enfants présentant un TDL d'âge scolaire parviennent à effectuer des généralisations de premier et de second ordre, c'est-à-dire d'apprendre une règle de catégorisation et de la généraliser, pour autant que cette règle soit simple et basée sur un seul critère de nature perceptive. Des différences de performance peuvent être notées entre les enfants plus jeunes et plus âgés. Nous avons proposé d'inscrire ces différences dans une perspective développementale et amené quelques pistes d'investigation. Il serait particulièrement intéressant d'observer dans quelle mesure ces résultats peuvent être répliqués sur de plus grandes cohortes. D'un point de vue clinique, enfin, s'intéresser aux variables et paramètres la maîtrise de ce type de règle par des enfants présentant un TDL plus âgés, malgré des écarts persistants en termes de scores aux épreuves de vocabulaire et l'observation de difficultés d'apprentissage de mots par ailleurs, permettrait d'ajuster les évaluations proposées et d'identifier les contextes plus ou moins facilitateurs pour calibrer plus adéquatement les interventions proposées. 


\section{Remerciements}

Nous remercions tous les enfants ayant participé à l'étude, ainsi que leurs parents ou tuteurs et enseignants pour leur implication dans le projet. Ce travail est soutenu par le fonds FRESH du F.R.S.-F.N.R.S. (réf. 6200320F).

\section{Bibliographie}

Aguilar, J. M., Plante, E., \& Sandoval, M. (2018). Exemplar Variability Facilitates Retention of Word Learning by Children With Specific Language Impairment. Language, Speech, and Hearing Services in Schools, 49(1), 72-84. https://doi.org/10.1044/2017_LSHSS-17-0031

Alt, M., \& Plante, E. (2006). Factors That Influence Lexical and Semantic Fast Mapping of Young Children With Specific Language Impairment. Journal of Speech, Language, and Hearing Research, 49(5), 941-954. https://doi.org/10.1044/1092-4388(2006/068)

Aslin, R. N. (2017). Statistical learning: A powerful mechanism that operates by mere exposure. Wiley Interdisciplinary Reviews: Cognitive Science, 8(1-2), e1373. https://doi.org/10.1002/wcs.1373

Aslin, R. N., \& Newport, E. L. (2012). Statistical Learning : From Acquiring Specific Items to Forming General

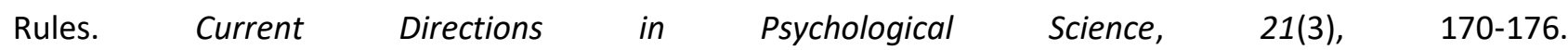
https://doi.org/10.1177/0963721412436806

Bishop, D. V. M., Snowling, M. J., Thompson, P. A., Greenhalgh, T., \& and the CATALISE-2 consortium. (2017). Phase 2 of CATALISE: A multinational and multidisciplinary Delphi consensus study of problems with language development: Terminology. Journal of Child Psychology and Psychiatry, 58(10), 1068-1080. https://doi.org/10.1111/jcpp.12721

Brooks, P. J., Maouene, J., Sailor, K., \& Seiger-Gardner, L. (2017). Modeling the Semantic Networks of SchoolAge Children with Specific Language Impairment and Their Typical Peers. In M. LaMendola \& J. Scott (Eds.), Proceedings of the $41^{\text {st }}$ annual Boston University Conference on Language Development (pp. 114-127). Cascadilla Press.

Charest, M., \& Skoczylas, M. J. (2019). Lexical Diversity Versus Lexical Error in the Language Transcripts of Children With Developmental Language Disorder: Different Conclusions About Lexical Ability. American Journal of Speech-Language Pathology, 28(3), 1275-1282. https://doi.org/10.1044/2019_AJSLP-18-0143

Collisson, B. A., Grela, B., Spaulding, T., Rueckl, J. G., \& Magnuson, J. S. (2015). Individual differences in the shape bias in preschool children with specific language impairment and typical language development: Theoretical and clinical implications. Developmental Science, 18(3), 373-388. https://doi.org/10.1111/desc.12219 
Conti-Ramsden, G., Durkin, K., Toseeb, U., Botting, N., \& Pickles, A. (2018). Education and employment outcomes of young adults with a history of developmental language disorder. International Journal of Language \& Communication Disorders, 53(2), 237-255.

Conti-Ramsden, G., St Clair, M. C., Pickles, A., \& Durkin, K. (2012). Developmental Trajectories of Verbal and Nonverbal Skills in Individuals With a History of Specific Language Impairment: From Childhood to Adolescence. Journal of Speech, Language, and Hearing Research, 55(6), 1716-1735. https://doi.org/10.1044/1092-4388(2012/10-0182)

Evans, J. L., Saffran, J. R., \& Robe-Torres, K. (2009). Statistical learning in children with specific language impairment. Journal of Speech, Language, and Hearing Research, 52(2), 321-335.

Gray, S. (2003). Word-Learning by Preschoolers With Specific Language Impairment : What Predicts Success? Journal of Speech, Language, and Hearing Research, 46, 56-67.

Gray, S. (2004). Word Learning by Preschoolers With Specific Language Impairment : Predictors and Poor Learners. Journal of Speech, Language, and Hearing Research, 47, 1117-1132.

Gray, S. (2006). The Relationship Between Phonological Memory, Receptive Vocabulary, and Fast Mapping in Young Children With Specific Language Impairment. Journal of Speech, Language, and Hearing Research, 49(5), 955-969. https://doi.org/10.1044/1092-4388(2006/069)

Haebig, E., Saffran, J. R., \& Ellis Weismer, S. (2017). Statistical word learning in children with autism spectrum disorder and specific language impairment. Journal of Child Psychology and Psychiatry, 58(11), 1251-1263. https://doi.org/10.1111/jcpp.12734

Im-Bolter, N., Johnson, J., \& Pascual-Leone, J. (2006). Processing Limitations in Children With Specific Language Impairment: The Role of Executive Function. Child Development, 77(6), 1822-1841. https://doi.org/10.1111/j.1467-8624.2006.00976.x

Jackson, E., Leitao, S., \& Claessen, M. (2016). The relationship between phonological short-term memory, receptive vocabulary, and fast mapping in children with specific language impairment: STM, receptive vocabulary and fast mapping in children with SLI. International Journal of Language \& Communication Disorders, 51(1), 61-73. https://doi.org/10.1111/1460-6984.12185

Jackson, E., Leitão, S., Claessen, M., \& Boyes, M. (2021). Word learning and verbal working memory in children with developmental language disorder. Autism \& Developmental Language Impairments, 6, 1-20. https://doi.org/10.1177/23969415211004109

Kan, P. F., \& Windsor, J. (2010). Word Learning in Children With Primary Language Impairment : A MetaAnalysis. Journal of Speech Language and Hearing Research, 53(3), 739-756. https://doi.org/10.1044/10924388(2009/08-0248) 
Kapa, L. L., \& Erikson, J. A. (2020). The Relationship Between Word Learning and Executive Function in Preschoolers With and Without Developmental Language Disorder. Journal of Speech, Language, and Hearing Research, 63(7), 2293-2307. https://doi.org/10.1044/2020_JSLHR-19-00342

Krzemien, M., Dauvister, E., \& Maillart, C. (2020). La généralisation chez les enfants avec un trouble développemental du langage : S'intéresser au niveau lexico-sémantique. A.N.A.E., 32(3).

Krzemien, M., Thibaut, J.-P., Jemel, B., Levaux, E., \& Maillart, C. (2021). How do children with developmental language disorder extend novel nouns? Journal of Experimental Child Psychology, 202, Article 105010. https://doi.org/10.1016/j.jecp.2020.105010

Lammertink, I., Boersma, P., Wijnen, F., \& Rispens, J. (2017). Statistical Learning in Specific Language Impairment: A Meta-Analysis. Journal of Speech, Language, and Hearing Research, 60(12), 3474-3486. https://doi.org/10.1044/2017_JSLHR-L-16-0439

Leonard, L. B. (2014). Children with Specific Language Impairment (2nd edition). The MIT Press.

Lum, J. A. G., Conti-Ramsden, G., Morgan, A. T., \& Ullman, M. T. (2014). Procedural learning deficits in specific language impairment (SLI) : A meta-analysis of serial reaction time task performance. Cortex, 51, 1-10. https://doi.org/10.1016/j.cortex.2013.10.011

Mainela-Arnold, E., \& Evans, J. L. (2014). Do statistical segmentation abilities predict lexical-phonological and lexical-semantic abilities in children with and without SLI? Journal of Child Language, 41(2), 327-351. https://doi.org/10.1017/\$0305000912000736

Mainela-Arnold, E., Evans, J. L., \& Coady, J. A. (2010). Explaining Lexical-Semantic Deficits in Specific Language Impairment : The Role of Phonological Similarity, Phonological Working Memory, and Lexical Competition. Journal of Speech, Language, and Hearing Research, 53(6), 1742-1756. https://doi.org/10.1044/10924388(2010/08-0198)

Mathôt, S., Schreij, D., \& Theeuwes, J. (2012). OpenSesame : An open-source, graphical experiment builder for the social sciences. Behavior Research Methods, 44(2), 314-324. https://doi.org/10.3758/s13428-0110168-7

McGregor, K. K., Oleson, J., Bahnsen, A., \& Duff, D. (2013). Children with developmental language impairment have vocabulary deficits characterized by limited breadth and depth: Vocabulary deficits. International Journal of Language \& Communication Disorders, 48(3), 307-319. https://doi.org/10.1111/1460-6984.12008

McMurray, B., Horst, J. S., \& Samuelson, L. K. (2012). Word learning emerges from the interaction of online referent selection and slow associative learning. Psychological Review, 119(4), 831-877. https://doi.org/10.1037/a0029872

Obeid, R., Brooks, P. J., Powers, K. L., Gillespie-Lynch, K., \& Lum, J. A. G. (2016). Statistical Learning in Specific Language Impairment and Autism Spectrum Disorder: A Meta-Analysis. Frontiers in Psychology, 7, Article 1245. https://doi.org/10.3389/fpsyg.2016.01245 
Pauls, L. J. \& Archibald, L. M. (2016). Executive Functions in Children With Specific Language Impairment : A Meta-Analysis. Journal of Speech, Language, and Hearing Research, 1-13.

Perry, L. K., Samuelson, L. K., Malloy, L. M., \& Schiffer, R. N. (2010). Learn Locally, Think Globally : Exemplar Variability Supports Higher-Order Generalization and Word Learning. Psychological Science, 21(12), 1894-1902. https://doi.org/10.1177/0956797610389189

Reeder, P. A., Newport, E. L., \& Aslin, R. N. (2013). From shared contexts to syntactic categories : The role of distributional information in learning linguistic form-classes. Cognitive Psychology, 66(1), 30-54. https://doi.org/10.1016/j.cogpsych.2012.09.001

Sheng, L., \& McGregor, K. K. (2010a). Lexical-Semantic Organization in Children With Specific Language Impairment. Journal of Speech Language and Hearing Research, 53(1), 146-159. https://doi.org/10.1044/1092-4388(2009/08-0160)

Sheng, L., \& McGregor, K. K. (2010b). Object and Action Naming in Children With Specific Language Impairment. Journal of Speech Language and Hearing Research, 53(6), 1704-1719. https://doi.org/10.1044/1092-4388(2010/09-0180)

Sloutsky, V. M., Deng, W., Fisher, A. V., \& Kloos, H. (2015). Conceptual influences on induction : A case for a late onset. Cognitive Psychology, 82, 1-31. https://doi.org/10.1016/j.cogpsych.2015.08.005

Smith, L. B. (2003). Learning to Recognize Objects. Psychological Science, 14(3), 244-250.

Smith, L. B., Jones, S. S., Landau, B., Gershkoff-Stowe, L., \& Samuelson, L. (2002). Object name learning provides on-the-job training for attention. Psychological Science, 13(1), 13-19.

Thordardottir, E. T., \& Weismer, S. E. (2002). Verb argument structure weakness in specific language impairment in relation to age and utterance length. Clinical Linguistics \& Phonetics, 16(4), 233-250. https://doi.org/10.1080/02699200110116462

Vugs, B., Cuperus, J., Hendriks, M., \& Verhoeven, L. (2013). Visuospatial working memory in specific language impairment: A meta-analysis. Research in Developmental Disabilities, 34(9), 2586-2597. https://doi.org/10.1016/j.ridd.2013.05.014 


\section{Liste des tableaux et figures}

Tableaux

Tableau 1.

Caractéristiques langagières et cognitives, par groupe

\begin{tabular}{|c|c|c|c|c|c|c|}
\hline & \multicolumn{2}{|c|}{ TDL } & \multicolumn{2}{|c|}{ CTRL } & \multirow[t]{2}{*}{$t(32)$} & \multirow[t]{2}{*}{$p$} \\
\hline & $M$ & $E T$ & $M$ & $E T$ & & \\
\hline Age (mois) & 112.00 & 19.28 & 113.94 & 18.14 & 0.30 & .764 \\
\hline QINV & 93.35 & 8.02 & 94.12 & 6.77 & 0.30 & .766 \\
\hline Word repetition & -9.03 & 9.48 & 0.77 & 0.62 & 4.25 & $<.001$ \\
\hline Designation (lexique) & 81.65 & 18.47 & 114.29 & 12.79 & 5.99 & $<.001$ \\
\hline Denomination & -1.20 & 1.47 & 0.16 & 0.76 & 3.38 & .002 \\
\hline Designation (syntaxe, erreurs) & -2.15 & 1.36 & -0.16 & 0.72 & 5.35 & $<.001$ \\
\hline Complétion d'énoncés & -3.26 & 1.21 & -0.19 & 0.73 & 8.96 & $<.001$ \\
\hline
\end{tabular}

Note. $\mathrm{TDL}=$ trouble développemental du langage, $\mathrm{CTRL}$ = enfants contrôle, QINV = QI non-verbal, ET = écarttype. Les scores sont exprimés en scores standardisés. 
Figures

Figure 1.

Description et Organisation des Stimuli.
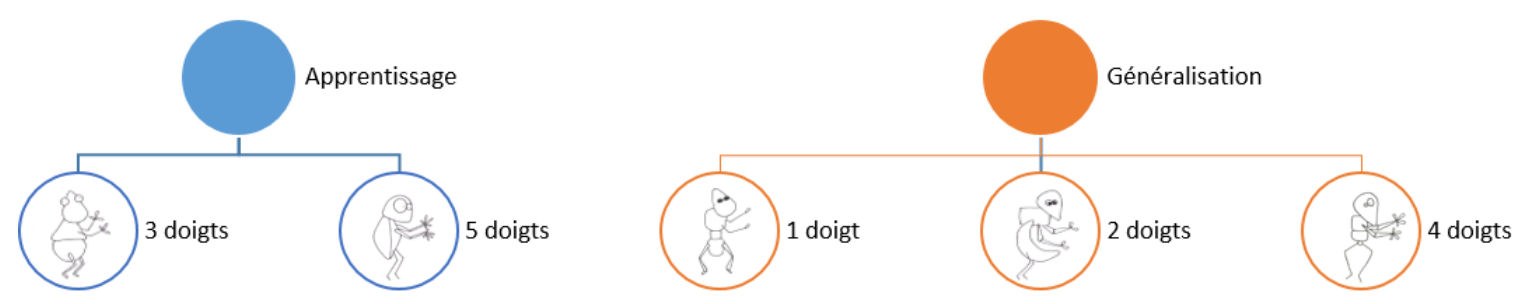

Note. L'apprentissage fait référence à la première phase de l'étude, la tâche de catégorisation. La généralisation fait référence à la seconde phase. Lors de cette seconde phase, de nouvelles catégories qui répondent à un critère pertinent de même type sont proposées.

\section{Figure 2.}

Illustrations des Phases d'Apprentissage et de Généralisation.

a.

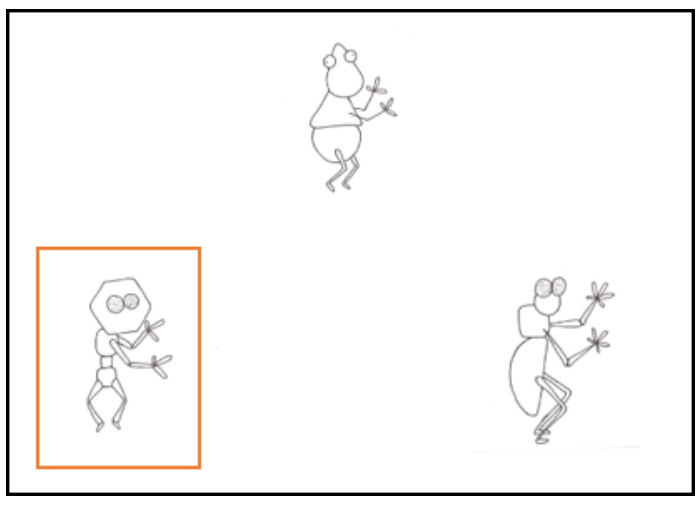


APPRENTISSAGE DE REGLES ET TDL

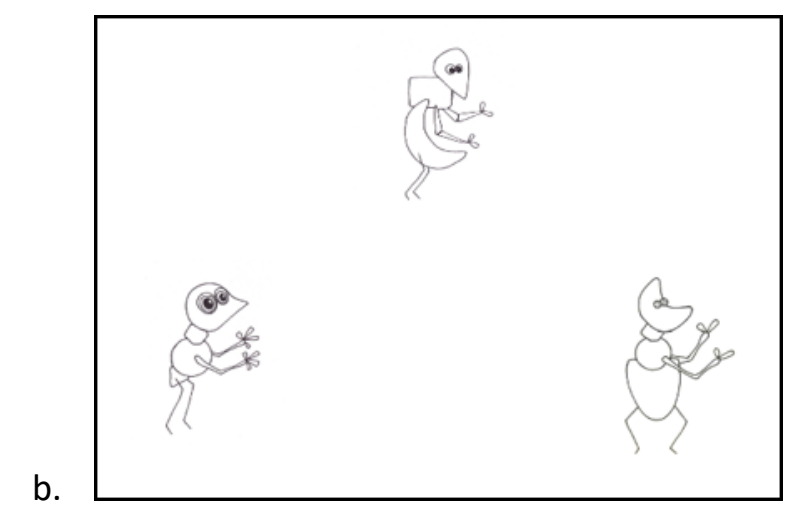

Note. La figure 2a représente une planche de la tâche de catégorisation, avec feedback en cas d'erreur. La figure $2 b$ représente une planche de la tâche de généralisation. 